\title{
EXPLOTACIÓN DEL SECTOR FORESTAL Y SU APORTE AL CRECIMIENTO ECONÓMICO DE HONDURAS EN EL PERIODO 2000-2012 ${ }^{1}$
}

\author{
ISRAEL E. HERNÁNDEZ \\ Facultad de Ciencias Económicas, Carrera de Economía \\ Universidad Nacional Autónoma de Honduras. \\ e-mail: enri.lee.littlem@hotmail.com \\ INGRID VELÁSQUEZ \\ Facultad de Ciencias Económicas, Carrera de Economía \\ Universidad Nacional Autónoma de Honduras. \\ DILMA VILLATORO \\ Facultad de Ciencias Económicas, Carrera de Economía \\ Universidad Nacional Autónoma de Honduras.
}

\section{RESUMEN}

La presente investigación tiene como finalidad detallar el comportamiento del sector forestal en la comercialización de bienes madereros en el periodo 2000-2012; de igual manera se pretende exponer el nivel de aporte que hace este sector al crecimiento económico del país, tomando estos datos del Instituto de Conservación y Desarrollo Forestal. La teoría que sustenta la investigación se basa en la teoría del desarrollo sostenible la cual ha sido desarrolladla por la Organización de las Naciones Unidas. El documento describe el comportamiento de las variables Crecimiento Económico, Silvicultura (dentro del sector primario) e Industria Forestal (dentro del sector secundario) en el que se expone cual ha sido su comportamiento durante el periodo, llegando a concluir que dicho sector aporta al crecimiento económico de Honduras un $0.87 \%$ presentando así un déficit en la balanza comercial de productos madereros durante los años en estudio; anudado a lo anterior en el país el aprovechamiento en cuanto al bosque se refleja a nivel privado con fines industriales utilizando la madera en rollo de pino en mayores proporciones, es decir el aprovechamiento ha sido únicamente de carácter de materia prima y pocos son los procesos tecnológicos que permiten a dichos productos obtener un mayor valor agregado. También se concluye que al ser este un sector poco significativo cuando se compara con otros de mayor desempeño un mayor crecimiento económico vendrá determinado por el desempeño de los diferentes

\footnotetext{
${ }^{1}$ Los manuscritos aceptados son Artículos en Prensa que han sido revisados por pares y aceptados para su publicación por el Consejo Editorial de esta revista. Todavía no han sido editados para su copia y / o formateados en el estilo de casa de publicación del IIES, y pueden no tener la funcionalidad completa del IIES, por ejemplo, es posible que los archivos suplementarios todavía deben añadirse, así como que los enlaces a referencias no funcionan aún, etc.. El texto podría todavía cambiar antes de la publicación final.

Aunque los manuscritos aceptados no tienen todos los detalles bibliográficos disponibles aún, ya pueden ser citados con el año de la publicación en línea de la siguiente manera: autor (s), título del artículo, la publicación (año). Por favor consulte estilo de referencia de la revista para el orden exacto de estos elementos, abreviatura de nombres de revistas y uso de puntuación.
} 
sectores y además del sector forestal; sin embargo los niveles de aporte del sector son poco significativos al PIB por su carácter de ser una industria primaria en la que el proceso productivo sigue siendo débil y desfasado. Es entonces que al obtener los resultados podemos afirmar que la volatilidad del sector no necesariamente generará cambios significativos al crecimiento del país.

Palabras clave: Sector Forestal, Productos Madereros, Crecimiento Económico, Explotación, Comercialización.

\title{
EXPLOITATION OF FORESTRY AND ITS CONTRIBUTION TO ECONOMIC GROWTH IN HONDURAS IN THE PERIOD 2000-2012
}

\author{
ISRAEL E. HERNÁNDEZ \\ Facultad de Ciencias Económicas, Carrera de Economía \\ Universidad Nacional Autónoma de Honduras \\ E-mail: enri.lee.littlem@hotmail.com \\ INGRID VELÁSQUEZ \\ Facultad de Ciencias Económicas, Carrera de Economía \\ Universidad Nacional Autónoma de Honduras \\ DILMA VILLATORO \\ Facultad de Ciencias Económicas, Carrera de Economía \\ Universidad Nacional Autónoma de Honduras
}

\begin{abstract}
This research aims to detail the behavior of the forestry sector in the commercialization of timber assets in the period 2000-2012; in the same way is intended to expose the level of contribution that makes this sector to the economic growth of the country, taking these data of the Institute of conservation and forestry development. The theory underpinning the research is based on the theory of sustainable development which has been developed it by the Organization of the United Nations. The document describes the behavior of the variables of economic growth, forestry (within the primary sector) and forestry (within the secondary sector) which sets out which has been their behavior during the period, coming to the conclusion that this sector contributes to the economic growth in Honduras a $0.87 \%$ thus presenting a deficit in the balance of trade of wood products over the years in study; knotted to the above in the country in terms of forest exploitation is reflected private for industrial purposes using wood in roll of pine in greater proportions, we can say that the exploitation has been only raw character and few are technological processes that allow these products to obtain a higher added value. It is also concluded that this being a sector little significant when compared with others of greater performance greater economic growth determined by the
\end{abstract}


performance of different sectors and in addition to the forest sector; However the levels of contribution of the sector are little significant GDP by its character of being a primary industry in which the production process is still weak and outdated. It is then that the results we can say that the volatility of the sector will not necessarily generate significant changes to the country's growth.

Keywords: forestry, wood products, economic growth, exploitation and marketing.

\section{INTRODUCCIÓN}

El sector forestal de Honduras forma parte de uno de las riquezas naturales más importantes que tiene el país. Por su ubicación geográfica Honduras presenta una ventaja natural que se ve plasmada en los recursos naturales que posee pues el país presenta un clima subtropical en las zonas bajas y templado en la zona montañosa, lo cual permite que se cree un ecosistema variado y abundante en recursos forestales; aproximadamente el $87 \%$ de la superficie territorial está catalogada como área de vocación forestal (Administración Forestal del Estado - Corporación Hondureña de Desarrollo Forestal [AFE-COHDEFOR], 1996).

El potencial de los recursos naturales debe ser tomado no sólo como parte de la belleza escénica que proporciona, sino también como un factor que influya de alguna manera en el crecimiento económico; llegando aun más lejos, debe ser tomado muy en cuenta cuando se habla de búsqueda del desarrollo económico del país; esto debido a la importancia que toma el sector forestal cuando se habla de producción, puesto que es aquí donde da inicio la actividad económica utilizando la dotación de recursos naturales como materia prima para el proceso de la creación de bienes que se comercializaran en la economía.

El sector forestal en Honduras se inicia y toma importancia por parte de las distintas organizaciones involucradas a este en los años 1960, en el que se definió como un sector de alta importancia y generador de crecimiento económico, pero lamentablemente no existió un consenso definido por parte de las autoridades hondureñas de propiciar el crecimiento sostenido del sector. Durante la década de 1974, se produjeron cambios en materia forestal por parte del Estado, estos cambios abarcaron un nuevo rumbo del sector pues el Estado paso a tomar tutela y asumió las medidas de estatización de todas las actividades forestales que conllevaban a la comercialización de los productos provenientes de los bosques, siendo anteriormente de la acción del Estado una actividad meramente basada en la iniciativa privada. Desde el periodo de 1974, la evolución del sector forestal en Honduras se ha caracterizado en cuanto a la disminución de su cobertura pues se han ejecutado acciones que perjudican de manera directa el sector y que abarcan acciones tales como el uso irracional del recurso, la degradación del ambiente, el desmejoramiento de la calidad de agua, etc. A 
esto se le une el incremento gradual de la demanda de productos derivados del bosque, que año tras año enfrenta extracciones de materias primas para ser comercializados buscando satisfacer la demanda de dichos bienes (AFE-COHDEFOR, 1996).

Dichas acciones mencionadas anteriormente, tienen un efecto en la producción de bienes que en este caso se consideran renovables y que su renovación es estrictamente a largo plazo. Es entonces que el incremento gradual de la demanda de bienes para consumo ha ocasionado una reducción drástica en el área boscosa del país, esto por motivos de expansión de las actividades agrícolas y en consecuencia se ha orientado a cambiar la vocación del recurso forestal hacia la utilización de los suelos para la actividad agraria y ganadera sin tener un proceso que sea eficaz para el establecimiento de áreas geográficas que estén destinadas a la producción agrícola sin reducir o perjudicar la calidad de los suelos forestales. Lo anterior debido a que el Gobierno no adoptó medidas ni reformas encaminadas a la sustentación equilibrada en el ámbito forestal, pues no existían leyes rígidas y concretas que permitieran hacer uso racional de los recursos naturales provenientes del bosque por parte de los involucrados a este (AFE-COHDEFOR, 1996).

Históricamente en Honduras el sector forestal ha contribuido de forma significativa al PIB. La producción de madera entre los años 1996-1998 presento un aporte casi de un 10\%, aunque en los últimos años este aporte ha disminuido. Adicionalmente se tiene de la industria y comercio forestal y de la leña un aporte como fuente del consumo enérgico del $70 \%$. El país es un importante exportador de madera aserrada, según AFE-COHDERFOR presentó un aporte a la economía de US\$ 45 millones para el año 2000, pero en realidad si se contabilizaran los servicios ambientales y sociales de los bosques, el papel del subsector en la economía nacional seria aun más evidente (Secretaría de Recursos Naturales y Ambiente [SERNA], 2005).

Un aspecto importante a destacar es que el aprovechamiento de este sector produce efectos con grandes externalidades, ya que los costos y beneficios privados son distintos que los costos y beneficios sociales, enfrentándose a dos escenarios, perjudicial para la sociedad que habita en el área, y beneficioso para el productor forestal. La mayor parte de los efectos negativos son responsabilidad de una serie de actividades económicas que compiten por el espacio con el bosque y en su avance lo destruyen o afectan su capacidad productiva futura (Pratt y Quijandria, 1997).

Según datos del Banco Central de Honduras (BCH, s.f.) para el periodo 2010-2012, la actividad económica mostro un comportamiento en términos reales de $3.8 \%$, esto determinado por una mayor demanda interna y externa de productos. La economía hondureña siguió presentando un comportamiento positivo en el año 2013, alcanzando niveles de crecimiento del producto interno bruto de $2.6 \%$, explicado por el mayor dinamismo de la 
agricultura, ganadería, caza, silvicultura, entre otras. En cuanto a dichas actividades se reflejo un crecimiento desacelerado de $4.0 \%$, esto debido variaciones en los precios internacionales.

"La importancia del recurso forestal en materia económica se evidencia en la participación del sector en el PIB agrícola que fluctúa entre 6 y 10\%" (Furones Fragoso, s.f., introducción, parra 3). Por su parte la balanza comercial del sector ha reflejado déficit anualmente a niveles de US\$ 88.8 millones, explicado especialmente en productos de madera y sus derivados (Secretaria de Agricultura y Ganadería [SAG], 2004).

El actual conflicto que presenta, es que a pesar de que el Estado ha generado una gran cantidad de políticas públicas dirigidas al fortalecimiento, cuidado y conservación del sector forestal, aun se presentan situaciones como la deforestación descontrolada y la tala ilegal de la madera en zonas protegidas que aún no están preparadas para ser explotadas. Esta situación ha venido siendo un factor negativo que no se ha podido controlar ni disminuir por parte de las autoridades competentes. Según Alviar Ramírez, Restrepo Patiño y Gallón Gómez (2002) las consecuencias de la tala indiscriminada de los bosques son más de carácter ecológico que económico, puesto que estas acciones conllevan a que se desencadena la pérdida de vida silvestre, flora, recursos hídricos etc. En cuanto a la explotación ilegal de madera, se define que "Ni siquiera la explotación ilegal de madera obtiene resultados favorables, pues la ilegalidad implica elevados costos por sobornos que reducen los márgenes de ganancia" (Alviar Ramírez et al., 2002, Introducción, parra. 1).

Para la medición del PIB por rama de actividad las actividades relacionadas con el sector forestal, son registradas en las cuentas nacionales, pero estas actividades están plasmadas dentro de las actividades de Agricultura, silvicultura caza y pesca. Lo anterior relacionado al sector primario. Además las cuentas nacionales registran algunas actividades forestales en la Industria de Manufactura, es decir en la fabricación de madera, muebles, corchos, papel y cartón, etc.; lo anterior se registra como sector secundario, es decir bienes transformados y uso final.

El sector forestal de Honduras ha generado un aporte al crecimiento económico del país, esto medido en la balanza comercial que año tras año muestra nivele de crecimiento no estable ni con una tendencia al alza en la comercialización de productos madereros. Las transacciones internacionales por medio de las exportaciones de madera de Honduras hacia otros países de la región mostraron que en el año 2007, 2008 y 2009 se exportaron US\$44.5 millones, US\$34.0 millones y US\$19.9 millones respectivamente. Cabe resaltar que las exportaciones de productos madereros son en calidad de insumos; pero el país también exporto en los años mencionados productos terminados provenientes del sector forestal definidos como distintos muebles de madera los cuales registraron montos de US\$26.6 millones, US\$22.5 millones y US\$5.6 millones (BCH, s.f.). De a cuerdo con lo anterior, se observa que Honduras está 
exportando mayores niveles de productos madereros como insumo y menores en calidad de productos terminados que tienen un mayor valor agregado.

Sin embargo, lo anterior permite ver que las actividades primarias forestales no registran algunas actividades de dicho sector pues la producción para el auto consumo rara vez es registrada en los informes de las instituciones competentes. Además de ello, no hay un registro formal de las actividades terciarias del sector forestal como el turismo derivado de los bosques, el transporte de los productos forestales, el gasto publico por parte del Estado para el cuidado y conservación de estos, investigación y desarrollo, tecnología, etc. Debido a lo anterior los niveles de explotación y comercialización pueden no ser expresados a su totalidad en los registros anuales.

Las causas principales del cambio de cobertura forestal en Honduras han sido la agricultura migratoria y expansión de ganadería, la población y colonización del bosque, la tenencia de la tierra, la dependencia de la leña para consumo energético, falta de coordinación entre políticas forestales y agrícolas, incendios forestales y los desastres naturales (Organización de las Naciones Unidas para la Alimentación y la Agricultura, [FAO], s.f.).

Considerando las situaciones anteriormente mencionadas resulta de gran importancia plantearnos la pregunta ¿Cuál es el aporte que ha ofrecido la explotación del sector forestal en el crecimiento económico de Honduras en el periodo 2000-2013?

\section{OBJETIVO DEL TRABAJO}

El objetivo de esta investigación es analizar la contribución del sector forestal al crecimiento económico de Honduras en el periodo 2000 a 2012 en el que se pretende Describir la evolución histórica del sector forestal, su comportamiento y su desempeño en el periodo 2000-2013; determinar cuáles son los productos provenientes de la actividad forestal y su nivel de explotación; cuantificar el nivel de importaciones y exportaciones de productos madereros y su comportamiento a lo largo del periodo y verificar la relación existente entre el crecimiento económico y el sector forestal.

\section{ASPECTOS METODOLOGICOS}

La investigación se desarrolla bajo un enfoque cuantitativo, basado en un tipo descriptivo, con el objeto de detallar el comportamiento del sector forestal en la comercialización de bienes madereros en el periodo 2000-2012, de igual manera exponer el nivel de aporte que hace este al crecimiento económico del país.

Así mismo la investigación tiene un diseño no experimental, longitudinal, con retrospectiva histórica. Utilizando series de tiempo que permitirán ver los cambios de las variables a través 
del tiempo en las que se va a recurrir a las variaciones de la exportación de productos madereros y los índices de crecimiento del Producto Interno Bruto.

Los datos a recolectar provendrán de fuentes secundarias, las principales fuentes de datos que se utilizan para realizar esta investigación son: Banco Central de Honduras $(\mathrm{BCH})$, Instituto Nacional de Conservación y Desarrollo Forestal, Áreas Protegidas y Vida Silvestre (ICF), de los años 2000-2012.

\section{PERSPECTIVA TEÓRICA}

La expresión desarrollo sostenible fue llevada al uso común por primera vez por la Comisión Mundial para el Ambiente y el Desarrollo, un grupo reunido por la Organización de las Naciones Unidas. La comisión hizo del desarrollo sostenible el tema de su informe final, Nuestro futuro común-, publicada en 1987. Ahí se define el termino como una forma de desarrollo o progreso que "satisface las necesidades del presente sin comprometer la capacidad de las generaciones venideras de satisfacer sus propias necesidades" (pp. 15-16).

De acuerdo con lo anterior, el desarrollo como tal y el crecimiento económico de los países, genera forzosamente distorsiones en los ecosistemas. Es por ello que al existir una interrelación entre todos los ecosistemas, el uso irracional de uno de estos afectara de manera directa a otro. En base a la teoría mencionada, la explotación del sector forestal deberá darse de manera sostenible, es decir que la tasa de agotamiento deberá ser menor a la tasa de regeneración forestal, de forma tal que el uso de los recursos naturales forestales no solo debe reflejar un beneficio colectivo para la sociedad sino también una conservación sostenida que permita la utilización y regeneración para el uso de los bosques. Es por ello que siendo el bosque un recurso renovable, debe buscarse un desarrollo sostenible que permita hacer de este recurso un generador de crecimiento y bienestar para todos los involucrados pues la tierra no debe ser degradada más allá de su recuperación razonable (World Commission on Environment and Development, s.f.).

En esencia, la teoría del desarrollo sostenible presenta un proceso de cambio en el que la explotación y aprovechamiento de los recursos forestales, el avance tecnológico y el cambio institucional aseguran un crecimiento sostenido que permita satisfacer a futuro las necesidades del país (World Commission on Environment and Development, s.f.).

\section{RESULTADOS}

\section{Evolución Histórica del Sector Forestal}

La evolución del sector forestal en el periodo 2000-2012 se ha enfrentado a muchos cambios en cuanto a su administración, teniendo en los primeros años de análisis a la AFE-CODEFOR 
como ejecutora de sus funciones. Es a partir del 2007 que cambia a la ICF por la reforma que se le da a la ley llamada Ley Forestal Áreas Protegidas y Vida Silvestre en el mismo año, cambiando así también su nominación pasando de ser un subsector a un sector en al país. Es de relevancia mencionar que el banco central de Honduras lo toma aun en la actualidad como un subsector puesto que según el Decreto No. 31-92 en el articulo 1 existe una relación directa entre el sector agrícola siendo este de mayor importancia del país en cuanto a su crecimiento por lo que en su desglose se presenta como subsector forestal por medio de silvicultura.

La participación del sector ha ido en aumento en cuanto a su cobertura a lo largo del periodo de estudio, así como el aprovechamiento que se le da a la madera en rollo de pino y latifoliado. En la figura $\mathrm{N}^{\circ} 1$ se observa que a lo largo del periodo es dominante en metros cúbicos la explotación de pino generando mayores rangos de cobertura en el 2002 y dando un obvio descenso a partir del 2006, contrario al aprovechamiento latifoliado que no ha tenido mayor aporte desde el 2001, manteniéndose constante hasta en la actualidad.

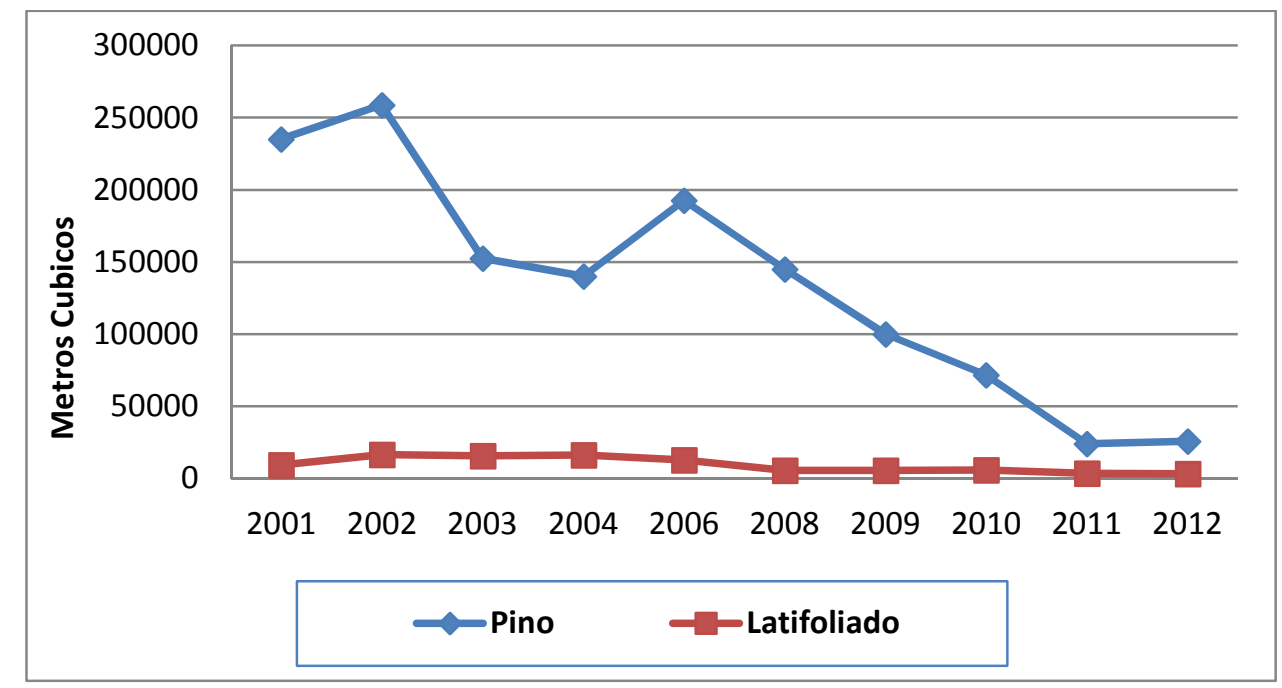

Figura 1. Honduras: Aprovechamiento de Madera en Rollo en M3, 2001-2012 (Fuente: Elaboración propia en base a datos de ICF)

En la figura $\mathrm{N}^{\circ} 2$ se observa que los fines de este aprovechamiento en las regiones se dan en el área industrial y domestica, presentado mayor volumen de participación en la industrial, esto tanto en el interior como en exterior del país con un 95\%, observándose que en el país el aprovechamiento en cuanto al bosque se refleja a nivel privado con fines industriales, utilizando la madera en rollo de pino en mayores proporciones. 


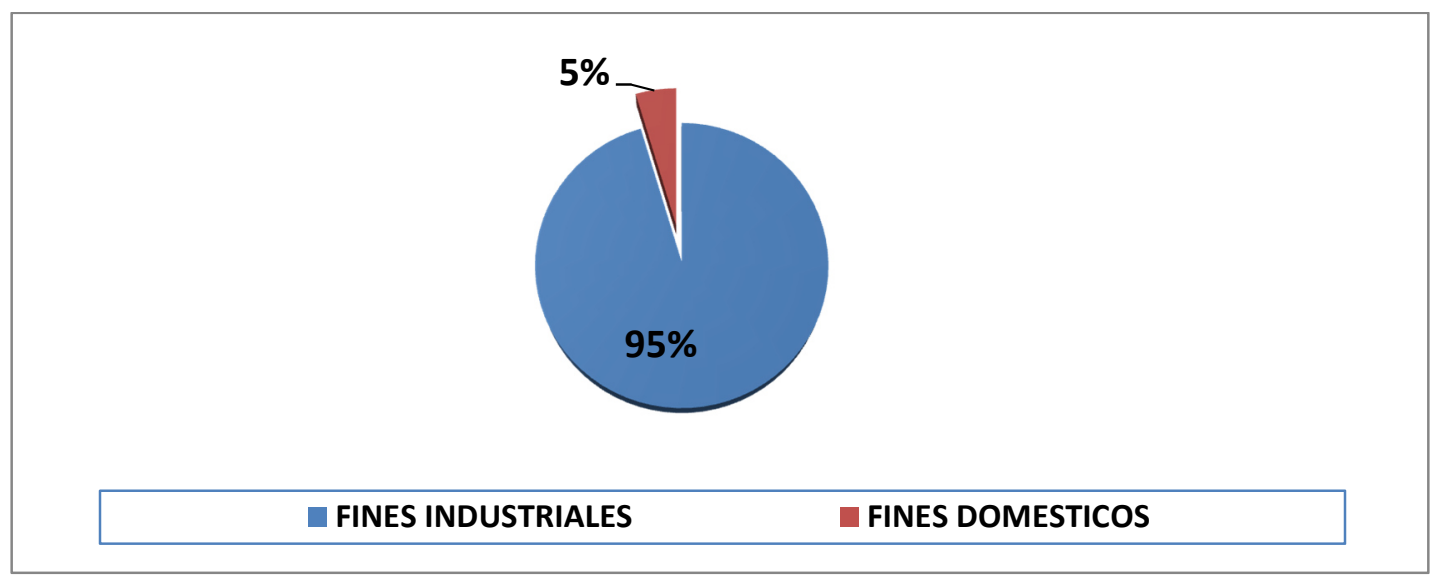

Figura 2. Honduras: Volumen de Madera en rollo aprovechado según finalidad $\left(\mathrm{m}^{3}\right)$, 2001-2012

(Fuente: Elaboración propia en base a datos de ICF)

En cuanto al aprovechamiento de tierra se puede mencionar que las regiones que más participación brindan al sector son Atlántida, Comayagua, El Paraíso, Francisco Morazán, La Mosquitia, Nor-Este de Olancho, Nor-Occidental, Occidente, Olancho, Pacifico, Rio Plátano y Yoro, esto a través del bosque nacional, ejidal y privado.

En la figura $\mathrm{N}^{\circ} 3$ se observa que dentro de estas regiones domina la participación del bosque privado en un $68 \%$, siguiéndole el nacional con un $23 \% \mathrm{y}$, en un porcentaje menor el ejidal con $9 \%$. Para el 2008, tomando este año como relevancia ya que en los años anteriores presentan comportamientos similares, entendiéndose como bosque nacional todo aquel que es protegido y explotado por el gobierno central a través de subastas públicas, los bosques privados son aquellos que tienen un dueño propio y que son protegidos a través de planes de manejos fomentados por el gobierno y el ejidal que es aquel tipo de bosque nacional que no puede ser utilizado para la agricultura.

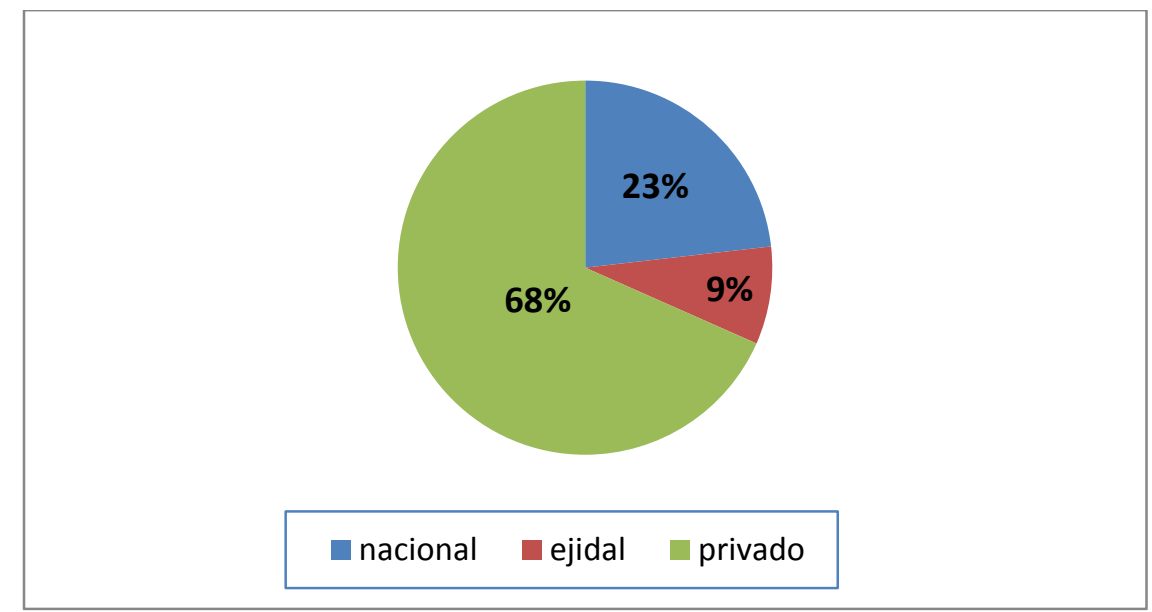

Figura 3. Honduras: Zonas de Producción de madera, 2001-2012 (Fuente: Elaboración propia en base a datos de ICF) 


\section{Productos Provenientes de la Actividad Forestal y su nivel de Explotación}

La actividad forestal en Honduras tiene relevancia en el proceso productivo de la creación de bienes para transformación y consumo. Los productos provenientes de la actividad forestal más comunes en el país son la madera en rollo (trozas), madera aserrada (timber, tablas y tablones), leña, carbón, resina de pino, resina de liquidámbar, astillas de ocote y postes para cerco.

A lo largo de los años la industria forestal primaria en Honduras ha venido presentando niveles de explotación de madera en rollo de pino relativamente mayor a la explotación de madera en rollo de latifoliado. En la figura $\mathrm{N}^{\circ} 4$ muestra un crecimiento de la explotación de madera de pino en los años 2000-2002 (medida en miles de metros cúbicos), una caída de los niveles de explotación en los años 2005-2012, desacelerándose de 920.2 a 385 miles de metros cúbicos; en cuanto a la explotación de madera proveniente de bosque latifoliado, los niveles de explotación han sido relativamente bajos y fluctuantes, es decir el aprovechamiento de este tipo de bosque ha tendido a ser mas errático año con año; los años 2003, 2005, 2008, 2010 y 2012 reflejaron los niveles de explotación más bajos.

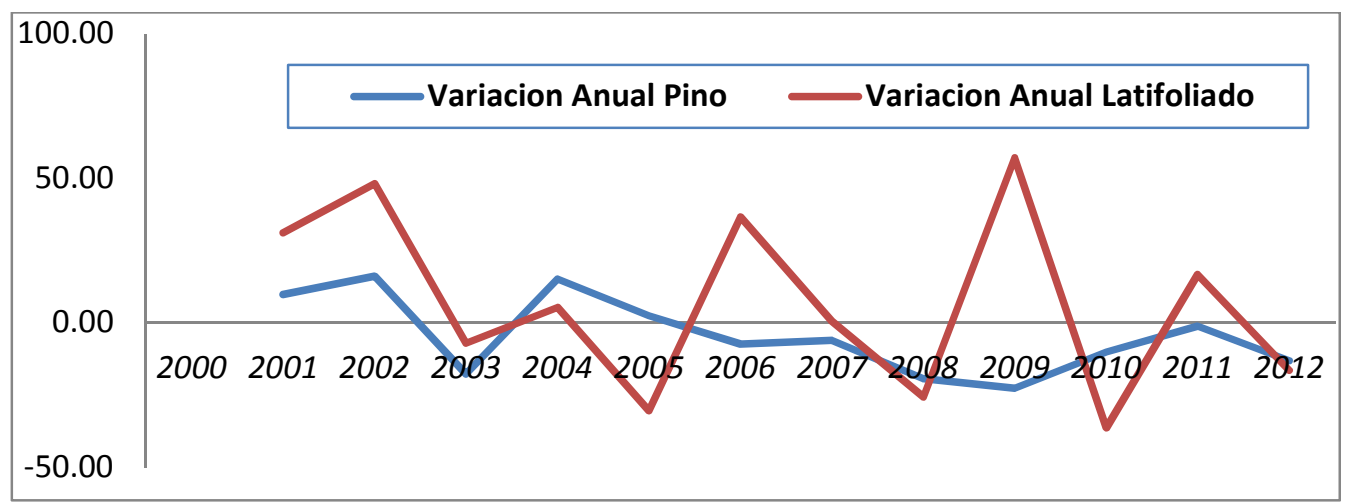

Figura 4. Honduras: Aprovechamiento de Madera en Rollo, Variación \%, 2000-2012 (Fuente: Elaboración propia en base a datos de ICF)

De estos aprovechamientos de madera en rollo, se desprende una serie de productos elaborados y semi-elaborados como ser: tablones, timber, tablas, vigas, palillos, forros, pisos y machimbre, los cuales a su vez son comercializados dentro del país y destinados al sector externo. Cabe mencionar que los productos más comunes que resultan del proceso de explotación del sector los más relevantes son: estacas de madera, madera aserrada latifoliada, madera aserrada de pino, muebles, postes de madera, puertas y ventanas, residuos de madera, resina y similares, semillas, tableros, palos de escoba, madera terciada, nasas, bolillos de madera, palillos de pino, bastones, carretes de madera, cajas de puros, tarimas de madera, plywood de madera, entre otros. 


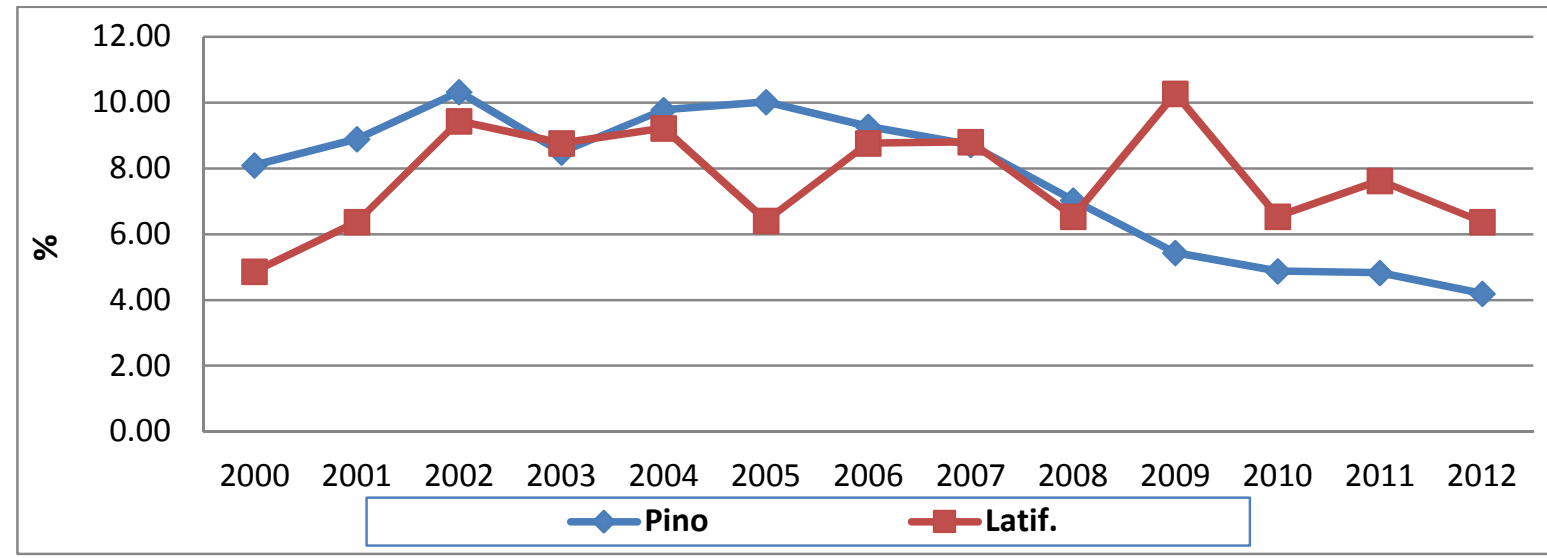

Figura 5. Honduras: Variación \% del Aprovechamiento de Madera en Rollo. Valores condensados, 2000-2012

(Fuente: Elaboración propia en base a datos de ICF)

Lo anterior plantea que los niveles de explotación de madera son considerablemente más significativos en cuanto al aprovechamiento de madera en rollo; la madera en rollo puede ser expresada como la materia prima que se obtiene de los árboles cortados y seccionados, es decir la grafica anterior puede mostrar el aprovechamiento de madera en rollo explotada como materia prima dentro del sector primario.

Otro de los productos provenientes del sector y que ha permitido formar parte de la actividad forestal es la fabricación de papel y cartón, esta actividad no se registra si no hasta los años 2008 y 2009. Los niveles de producción de papel y cartón son significativos pero cabe resaltar que este producto solo se registra en la balanza comercial forestal.

\section{Nivel de Importaciones y Exportaciones de Productos Madereros y su Comportamiento a lo Largo del Periodo}

A lo largo del periodo, el nivel de importaciones y exportaciones de los diferentes productos madereros (estacas de madera, madera aserrada, latifoliada, madera aserrada de pino, muebles, postes de madera, cartón y papel, puertas y ventanas, residuos de madera y tableros) en el sector forestal ha presentado un déficit en la balanza comercial. En los años 2006, 2007 y 2009 el déficit comercial alcanzo los US\$192,436,827, US\$215,116,070 y US\$190,852,625 respectivamente; no así para los años 2010, 2011 y 2012 donde los niveles de déficit comercial resulto ser poco significativo (US\$5.6, US\$16 y US\$2.5 millones respectivamente).

La figura $\mathrm{N}^{\circ} 6$, muestra el comportamiento de la balanza comercial de productos madereros durante el periodo de estudio; en lo sucedido en el año 2006, se observa que en cuanto a las exportaciones se obtuvo un aumento de casi US\$25 millones respecto a 2004, cabe mencionar que no se obtuvieron datos para el año 2005. En el año 2007 se observa un descenso en el nivel de exportaciones comparado al 2006 (US\$4, 935,613 menos para 2007) 
esto provocado por fenómenos de plagas forestales en bosques de pino e incendios forestales ocurridos en dichos años. En el año 2008, las exportaciones ascendieron a US\$73,120,436 (US\$22,271,476 mas que en 2007) esto debido a la mayor demanda de productos para su exportación. Con respecto al déficit comercial del año en mención la variación respecto a 2007 alcanzo el 52.3\% de déficit a pesar de haber reflejado una reducción en las importaciones de 2008 con respecto a 2007 de $34 \%$.

Asimismo la figura $\mathrm{N}^{\circ} 6$, muestra que para el 2009, la balanza comercial reflejo valores deficitarios puesto que las importaciones excedieron a las exportaciones en US\$190,852,625. Los años 2010, 2011 y 2012 reflejaron una reducción en la balanza comercial pues a pesar de que también existió déficit comercial estos ascendieron hasta US\$5,550,256; US\$16,150,063 y US\$2,479,446 respectivamente (valores negativos).

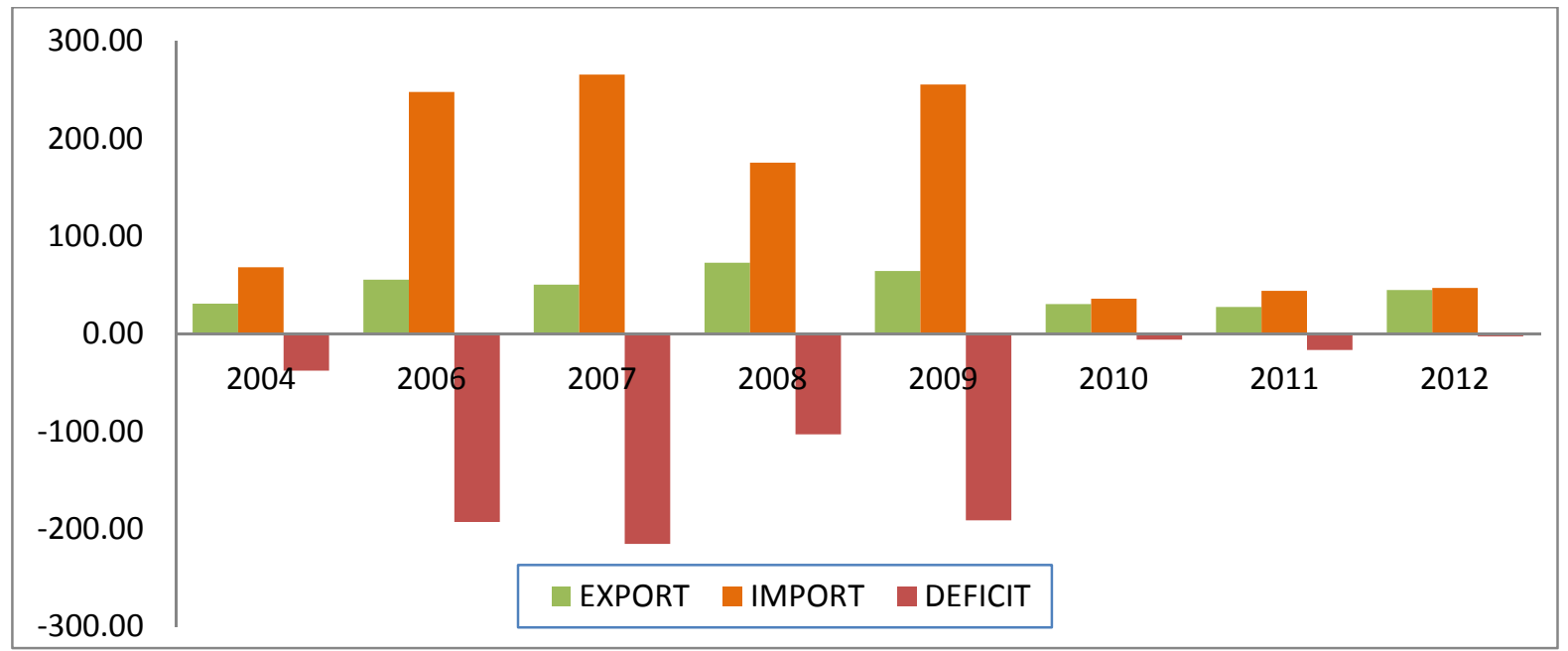

Figura 6. Honduras: Balanza Comercial del Sector Forestal (productos de madera), En millones de US\$ 2000-2012

(Fuente: Elaboración propia en base a datos de ICF)

La figura $\mathrm{N}^{\mathrm{o}}$, muestra el comportamiento de diferentes productos madereros primarios y secundarios durante el periodo 2005-2008. Los muebles, la madera aserrada de pino y latifoliado han provocado un superávit en la balanza comercial, mientras que los productos como papel y cartón han reflejado niveles de importación de US\$ 258, 070,750. Cabe mencionar que no se proporcionaron datos de exportación en cuanto a Papel y Cartón. 


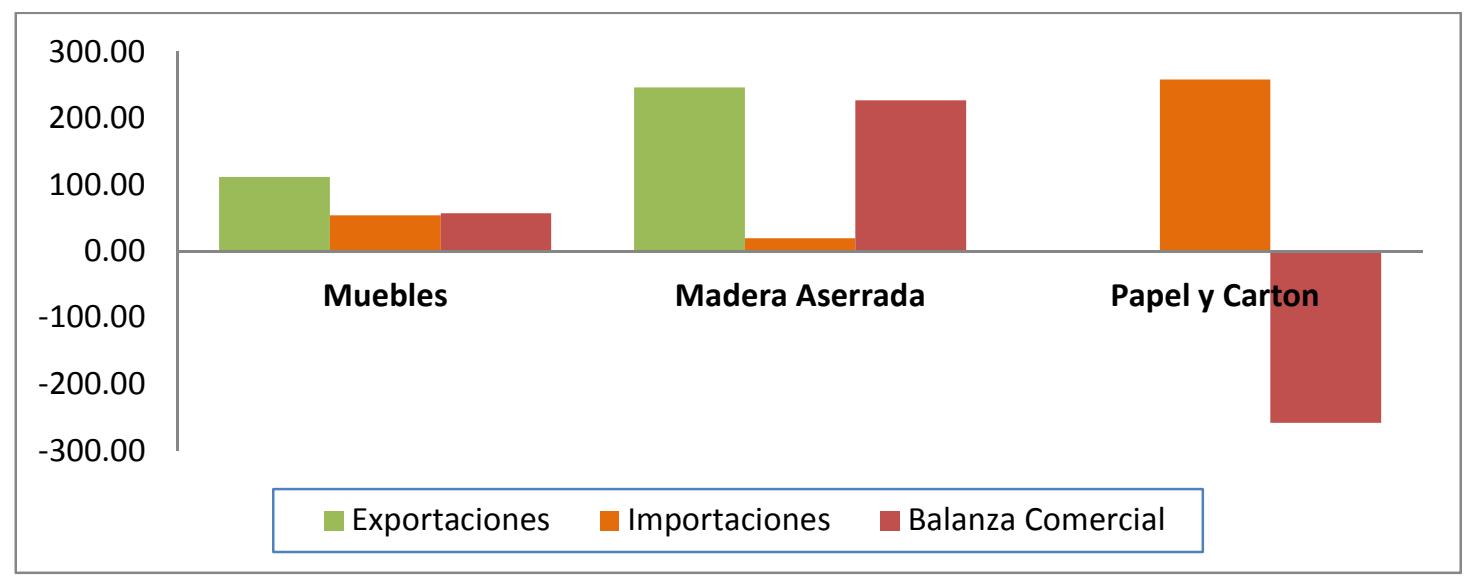

Figura 7. Honduras: Balanza Comercial de Productos Madereros. En millones de US\$, 2005-2008 (Fuente: Elaboración propia en base a datos de ICF)

La figura $\mathrm{N}^{\circ} 8$, refleja los mismos productos para el posterior periodo. Los productos Muebles reflejaron una caída de las exportaciones en US\$89,462,270 respecto al periodo anterior y un incremento acelerado de las importaciones que ascendió hasta US\$51, 798,660 respecto al periodo anterior. Lo anterior explicado por los diferentes acontecimientos que suscitaron durante el periodo tales como: la crisis política interna y los precios internacionales. En cuanto a Papel y Cartón se exportaron únicamente US\$40,447,488 e importaron US\$221,642,208 lo cual genero un déficit de US\$181,194,720. Por su parte la madera aserrada no obtuvo un resultado positivo con respecto al periodo anterior puesto que el déficit (que anteriormente resulto en superávit) alcanzo los US\$36, 560,093.

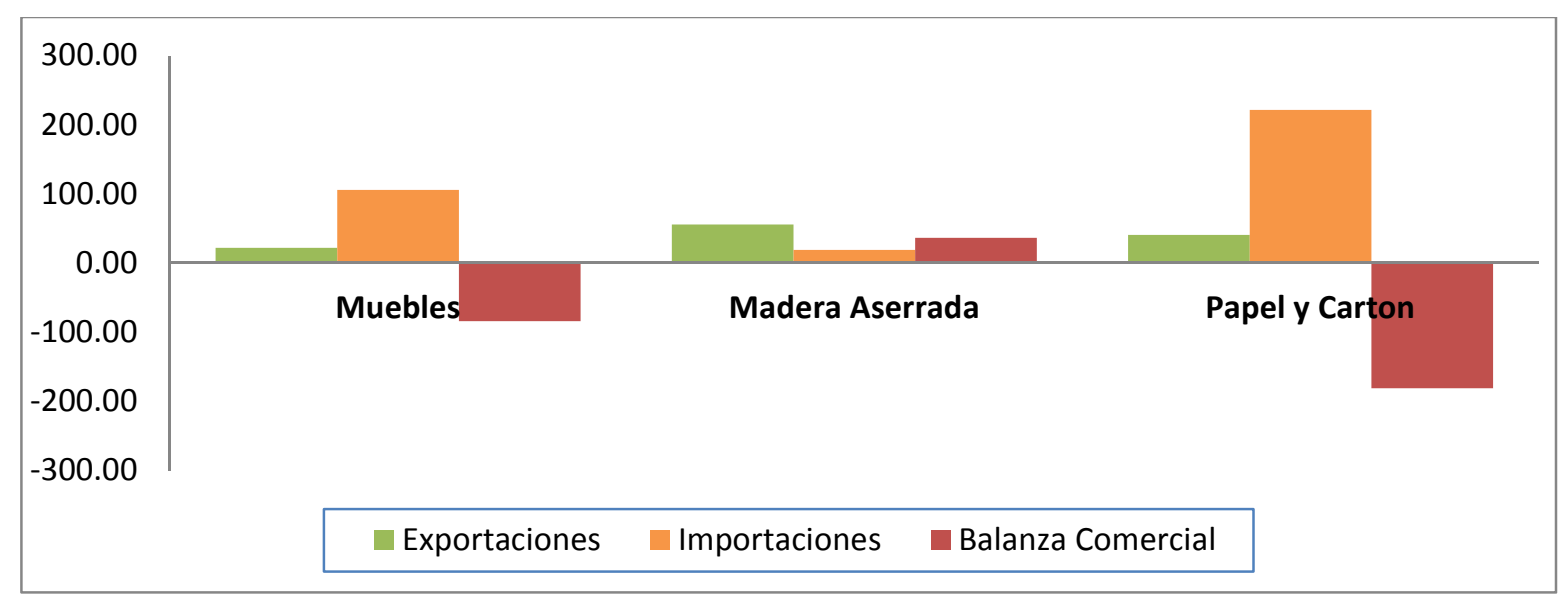

Figura 8. Honduras: Balanza Comercial de Productos Madereros. En millones de US\$, 2009-2012 (Fuente: Elaboración propia en base a datos de ICF)

\section{Relación Entre el Crecimiento Económico y el Sector Forestal}


La actividad forestal en la economía hondureña está planteada dentro de dos sectores, en el primario es la actividad económica llamada silvicultura y en el secundario es la actividad llamada Producción de Madera y Fabricación de Productos de Madera (ICF, 2013). Es de relevancia mencionar que el sector ha presentado una serie de cambios a lo largo de los años pasando de ser un subsector coordinado por la AFE-CODHEFOR a un sector manejado por el ICF teniendo mayor independencia en cuanto a su aporte a la economía del país.

El análisis de este sector es importante puesto que gracias a este se obtiene un porcentaje de aporte al crecimiento económico. La figura $\mathrm{N}^{\circ} 9$ refleja la relación existente entre la silvicultura y el PIB; evidenciando la existencia de una tendencia similar, presentando en ambos casos una baja en el 2009, esto por la crisis que se dio en el país en ese momento, teniendo en los siguientes años una mejoría continua a lo largo del tiempo.

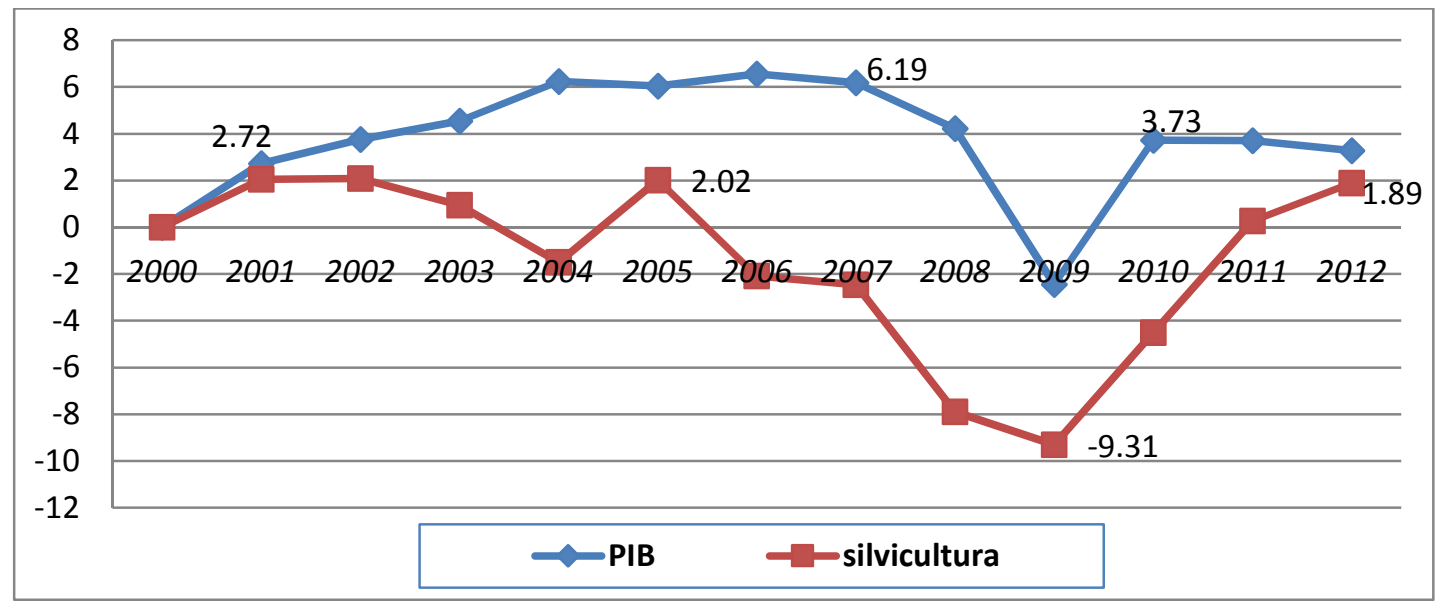

Figura 9. Honduras: Relación de la Silvicultura respecto al PIB, Variaciones \%, 2000-2012 (Fuente: Elaboración propia en base a datos de ICF)

Se observa que el sector empezó a desacelerarse de 2002 a 2004, (no así para 2005) y de 2006 a 2009 fueron los niveles de variación más negativos del periodo; a partir de allí el ritmo del sector se ha recuperado alcanzando hasta aproximadamente un crecimiento de $2 \%$. Es de hacer notar que el desempeño que ha tenido la actividad forestal en el sector primario ha disminuido a lo largo del tiempo obteniendo variaciones negativas para algunos años y positivas (bajas) para otros.

Por su parte la figura $\mathrm{N}^{\circ} 10$ muestra la relación entre la industria forestal (sector secundario) y el crecimiento obtenido en el país a lo largo del periodo de análisis, destacando que existen diferencias a grandes proporciones en cuanto al desempeño de la industria forestal, no así para el PIB que ha mostrado mantener una tendencia más constante que de la industria forestal (a diferencia de 2009). Asimismo se observa que la industria forestal ha mostrado 
una desaceleración drástica en el año 2007 y presenta una mejoría a partir de 2010 respecto al PIB. Esta actividad se ha enfrentado a grandes retos, el crecimiento en la industria manufacturera es uno de ellos pues la inversión en este rubro se hace relativamente más fácil por el tiempo de recuperación, lo cual no significa que se ha dejado de lado al sector secundario en la producción y fabricación de productos de madera, sin embargo es necesario mayores incentivos que propicien el dinamismo del sector para que lo lleven a mayores tasas de aporte al crecimiento económico del país.

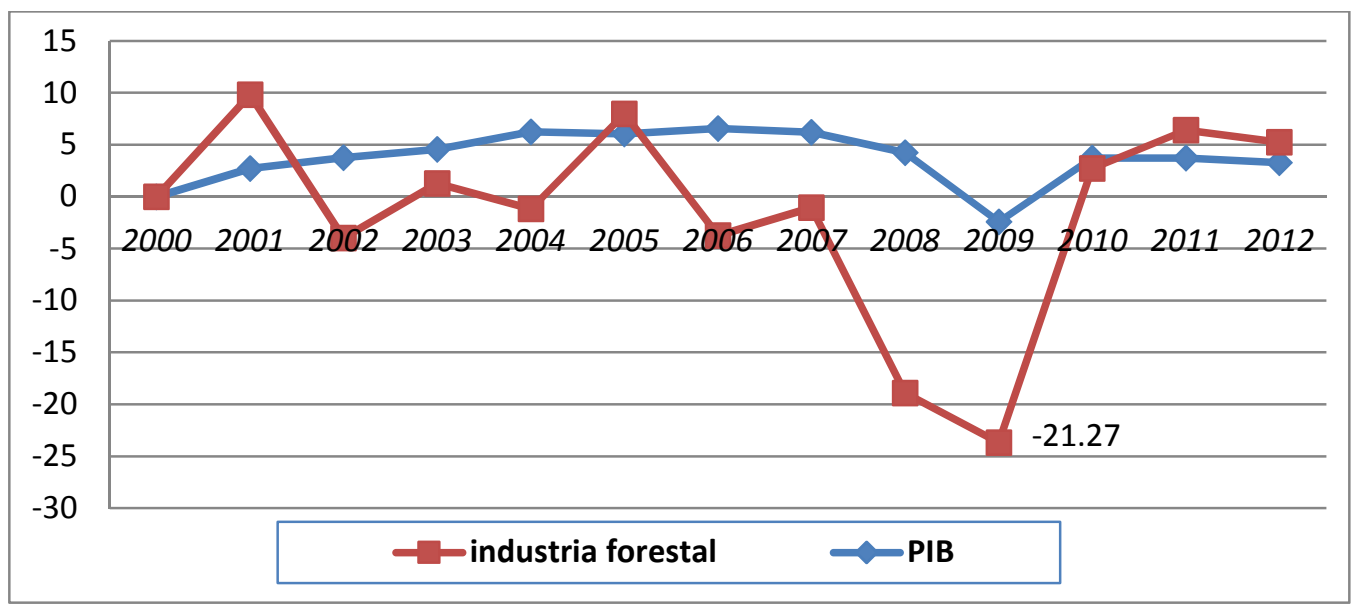

Figura 10. Honduras: Relación de la Industria Forestal Respecto al PIB, Variaciones \%, 2000-2012 (Fuente: Elaboración propia en base a datos de ICF)

En la figura $\mathrm{N}^{\circ} 11$ se observa la evolución que ha tenido en su conjunto el sector forestal tanto en la silvicultura como en la industria, y el aporte que este sector le ha dado al PIB hondureño, se destaca que tanto la silvicultura como la Industria forestal y el PIB tienden a responder de manera directa a cambios en el periodo; sin embargo la industria forestal tiende a absorber los efectos negativos más que proporcional a la silvicultura dado que se ha demostrado que dicha industria tiende a ser vulnerable a los efectos económicos directos e indirectos.

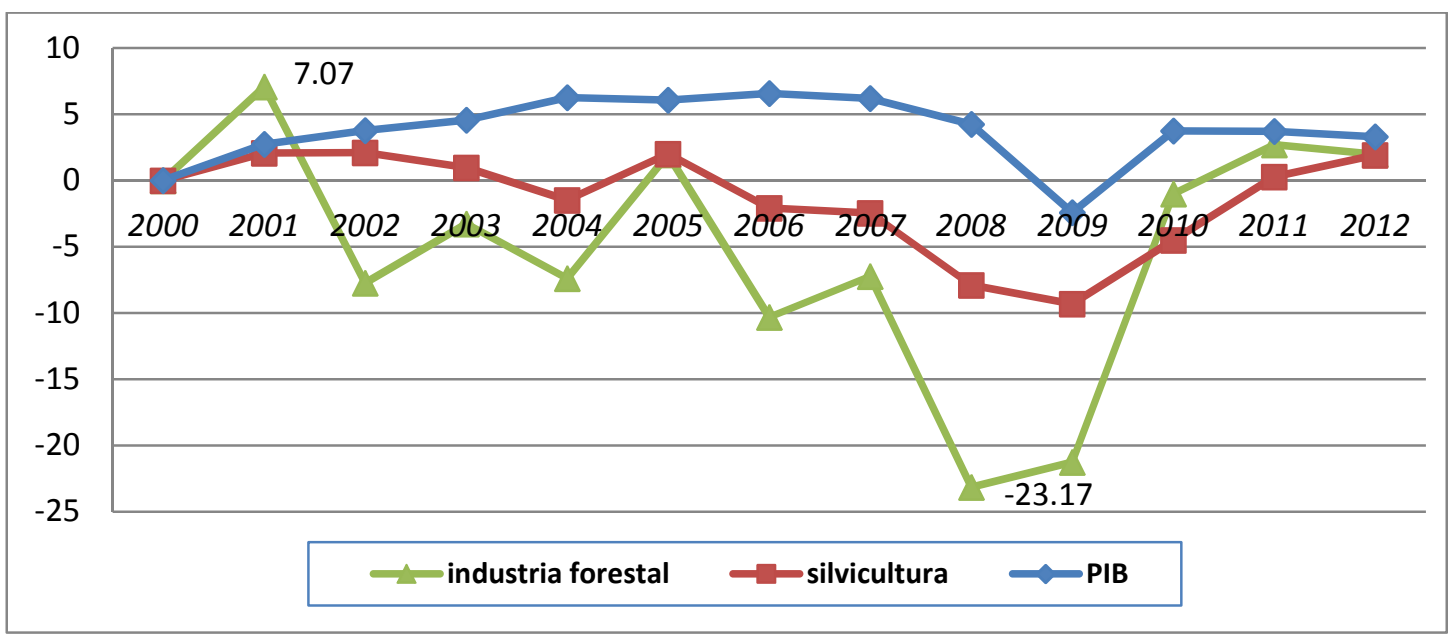

Figura 11. Honduras: Evolución del Sector Forestal, Variaciones \%, 2000-2012 
(Fuente: Elaboración propia en base a datos de ICF)

A pesar de que Honduras cuenta con todo el potencial para disfrutar de mayores ingresos derivados de la actividad forestal, por la condición en la que se encuentra y las características forestales favorables que posee, es de notar, que no se están obteniendo los resultados esperados, enfrentándose a porcentajes cada vez más bajos, teniendo una evolución y desempeño inconstante con un futuro incierto en los próximos años.

\section{Relación del Sector Forestal y el Empleo}

En el año 2007 el sector forestal empleo 687,879 hombres y 57,779 mujeres como empleos directos generando un total de 745,658 empleos.

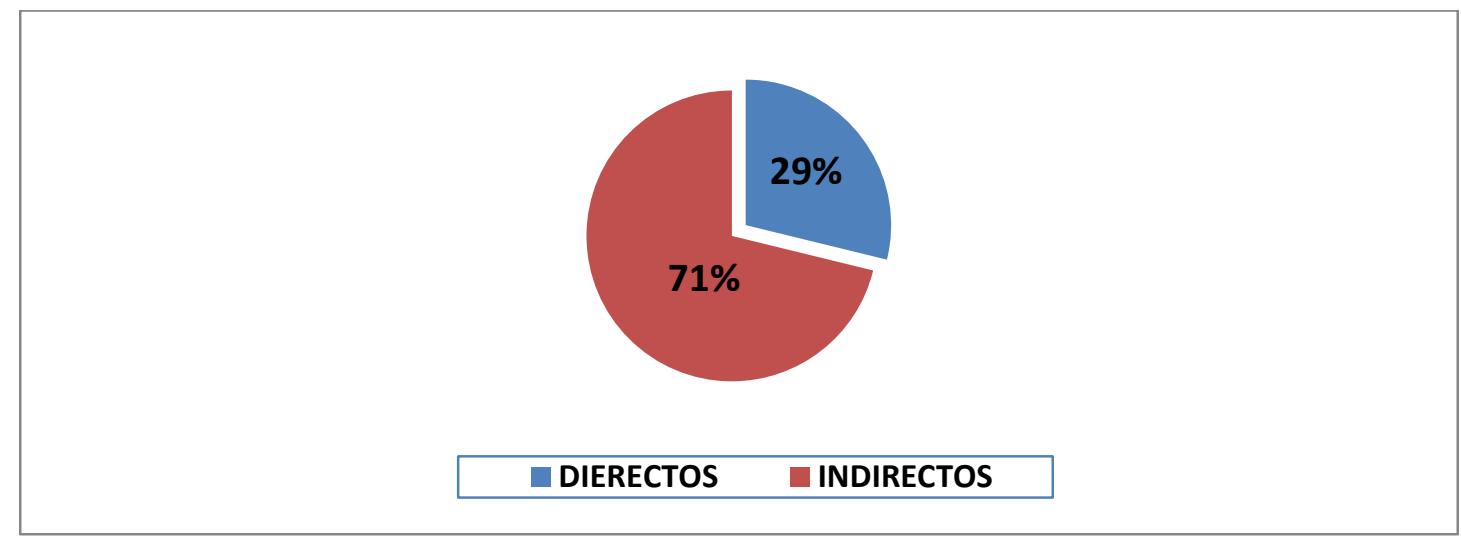

Figura 12. Honduras: Generación de empleo del Sector Forestal, En 2010

(Fuente: Elaboración propia en base a datos de ICF)

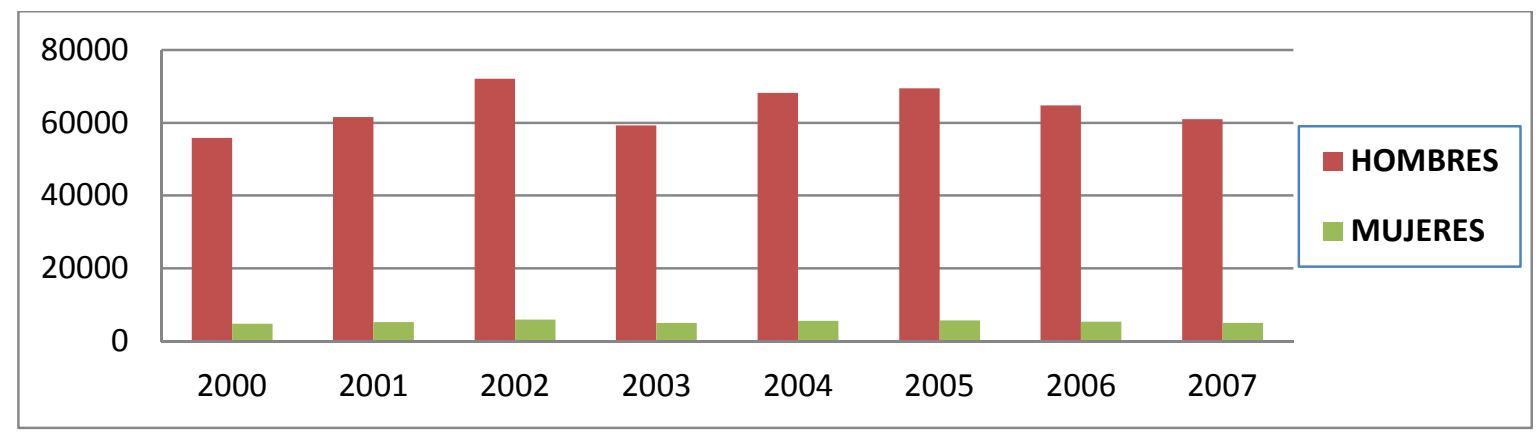

Figura 13. Honduras: Generación de empleo del sector forestal, 2000-2007

(Fuente: Elaboración propia en base a datos de ICF)

En la figura $\mathrm{N}^{\mathrm{o}} 13$, se aprecia que por el tipo de actividad en la que se utiliza mayor esfuerzo físico predomina la mano de obra masculina laborando en este sector. La tendencia se da al alza en los años 200-2002; no así en los años 2004-2007 donde la se mostro una desaceleración en el numero de empleos dentro del sector lo que prácticamente mostro una variación poco significativa al comparar los años 2000 y 2007. El análisis de esta figura refleja que el sector presenta fluctuaciones trascendentales en el empleo, con tendencias 
crecientes en periodos de tiempo y desaceleradas en otros; cabe destacar que este comportamiento se muestra en el empleo de hombres y, no así en el de las mujeres donde se aprecia una tendencia aunque poco significativa constante.

Es importante mencionar que no existen datos por parte de ICF que proporcionen el aporte al nivel de empleos indirectos de la actividad forestal, sin embargo se deduce que los empleos directos por ser más fáciles de contabilizar, son mayores que los empleos indirectos.

\section{CONSIDERACIONES FINALES}

1. El PIB mide la actividad económica, la que es de amplia utilización, pero tiene el defecto de no tomar en cuenta los daños ocasionados al ambiente. Por ende resulta dificultoso llegar a una medición exacta y correcta de la explotación del sector forestal y su aporte al crecimiento económico, pues los registros exponen únicamente la comercialización, transformación y comercio exterior, dejando fuera otros beneficios que ofrece el sector. Es entonces que las instituciones deben desagregar esta actividad de otras para expresar de manera más contundente cual es el nivel de aporte al crecimiento económico, pues si se tomasen datos como el costo de la excesiva deforestación, los beneficios del uso de un bosque para fines recreativos y en ocasiones la producción para el auto consumo; su aporte al PIB podría ser mucho más significativo.

2. El aporte de la actividad forestal al PIB es de $0.87 \%$ durante el periodo de estudio. Se llego al resultado anterior a través de la desagregación y sumatoria de la actividad de la industria forestal y la silvicultura representando estas el sector secundario y primario respectivamente, dividido entre la sumatoria total del PIB del periodo d estudio.

3. Las actividades forestales corresponden a las relacionadas más directamente con el bosque, por lo que en su mayor parte ésta se conforma por las actividades del sector primario (silvicultura), es decir, actividades en las que no se utiliza un continuo proceso de transformación que permita generar valor agregado de dichos producto pues los niveles de comercialización de madera aserrada de pino son relativamente mayores a los que se transforman en producto final.

4. El sector forestal hondureño en el periodo 2000-2012 ha reflejado una evolución inconstante presentando un comportamiento cada vez más a la baja, mostrando mayor volumen de participación en la industria forestal que la industria domestica, se observó que en el país el aprovechamiento en cuanto al bosque se refleja a nivel privado con fines industriales en donde se esta utilizando la madera en rollo de pino en mayores proporciones; la cobertura se refleja en las regiones de las cuales se obtienen los mayores beneficios en 
cuanto aprovechamiento forestal y la influencia de esta se ve en los diferentes tipos de bosques que dominan el país como ser el nacional, ejidal y privado.

\section{REFERENCIAS}

Administración Forestal del Estado-Corporación Hondureña de Desarrollo Forestal. (1996). Plan de acción forestal PLANFOR 1996-2015 (volumen I). Tegucigalpa Honduras: Graficentro.

Alviar Ramírez, M., Restrepo Patiño, M. y Gallón Gómez, S. (2002). Un modelo RSDAIDS para las importaciones de madera de Estados Unidos y sus implicaciones para Colombia. Recuperado el 19 de junio de 2014, de http://www.udea.edu.co/portal/page/portal/bibliotecaSedesDependencias/unidadesA cademicas/FacultadCienciasEconomicas/ElementosDiseno/Documentos/Borradores Economia/BorradCIE 3.pdf

Banco Central de Honduras. (s.f.). Memoria 2013. Recuperado el 15 de julio de 2014, de http://www.bch.hn/download/memoria/memoria_anual_2013.pdf

Furones Fragoso, L. (s.f.). Análisis nacional sobre gobernanza y cumplimiento de la legislación en el sector forestal en Honduras. Recuperado el 15 de julio de 2014, de http://siteresources.worldbank.org/EXTFORESTS/Resources/985784$\underline{1217874560960 / \text { AnalysisHonduras.pdf }}$

Organización de las Naciones Unidas para la Alimentación y la Agricultura. (s.f.). Principales causas del cambio de cobertura forestal. Conclusiones generales. Recuperado el 09 de julio de 2014, de http://www.fao.org/docrep/007/ac768s/AC768S03.htm

Pratt, L. y Quijandria, G. (1997). El sector forestal en Honduras: análisis de Sostenibilidad. Recuperado 14 de junio de 2014, de https://www.incae.edu/ES/clacds/publicaciones/pdf/cen740.pdf

Secretaria de Agricultura y Ganadería. (2004). Programa nacional forestal PRONAFOR Honduras 2004-2021. Tegucigalpa, Honduras: Agenda Forestal Hondureña. Recuperado el 15 de julio de 2014, de http://agendaforestal.org/documentos/PRONAFOR_documento.pdf

Secretaría de Recursos Naturales y Ambiente. (2005). Informe del estado y perspectivas del ambiente: Geo Honduras 2005. Tegucigalpa, Honduras: Programa de las Naciones 
Artículo Aceptado

Unidas para el Medio Ambiente. Recuperado el 14 de julio de 2014, de http://www.pnuma.org/deat1/pdf/GEOHonduras2005.pdf

World Commission on Environment and Development. (s.f.). Towards Sustainable Development. En Our common future (Chapter 2). Recuperado el 09 de julio de 2014, de http://www.un-documents.net/ocf-02.htm\#I 